\title{
Whole-body computed tomography is safe, effective and efficient in the severely injured hemodynamically unstable trauma patient
}

C open access

Citation: Ordoñez CA, Parra MW, Holguín A, García C, Guzmán RM, Padilla N, Caicedo Y, Orlas C, García A, Rodríguez HF, Serna JJ, Serna C. Whole-body computed tomography is safe, effective and efficient in the severely injured hemodynamically unstable trauma patient. Colomb Med (Cali).2020; 51(4) e-4054362

http://doi.org/10.25100/cm.v51i4.4362

Received : 08 Jul 2020

Revised : 09 Aug 2020

Accepted : 26 Sep 2020

Published: 29 Nov 2020

Keywords:

Whole-body; organ-selective CT; damage control trauma Care; Hemodynamic instability; trauma centers; advanced trauma life support care; X-Rays; • focused assessment with sonography for trauma; vascular system injuries; operating rooms; consensus.

Palabras clave:

TAC corporal total, TAC órgano selectivo, control de daños en trauma, inestabilidad hemodinámica; centros de tráuma; atención de soporte vital avanzados para traumatismos; rayos $x$; evaluación enfocada con ecografía para traumatismos; lesiones del sistema váscular; quirófanos; consenso.

\section{La Tomografía Computarizada Corporal Total es una herramienta segura, efectiva y eficiente en el paciente politraumatizado con inestabilidad hemodinámica}

\author{
Carlos A. Ordoñez ${ }^{1,2,3}$ Michael W. Parra ${ }^{4}$, Alfonso Holguín ${ }^{5}$ Carlos García ${ }^{5}$ Mónica \\ Guzmán-Rodríguez $^{6}$,Natalia Padilla ${ }^{7}{ }^{\infty}$,Yaset Caicedo ${ }^{7}$ D. Claudia Orlas ${ }^{8,9}$, Alberto

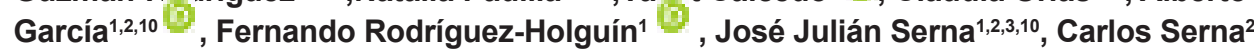 \\ ordonezcarlosa@gmail.com, carlos.ordonez@fvi.org.co
}

1 Fundación Valle del Lili, Department of Surgery, Division of Trauma and Acute Care Surgery. Cali, Colombia. 2 Universidad del Valle, Department of Surgery, Division of Trauma and Acute Care Surgery. Cali, Colombia. 3 Universidad Icesi, Cali, Colombia. 4 Broward General Level I Trauma Center, Department of Trauma Critical Care, Fort Lauderdale , FL, USA, 5 Fundación Valle del Lili, Department of Radiology. Cali, Colombia. 6 Universidad de Chile, Facultad de Medicina, Instituto de Ciencias Biomédicas, Santiago de Chile, Chile. 7 Fundación Valle del Lili, Centro de Investigaciones Clínicas (CIC), Cali, Colombia, 8 Brigham \& Women's Hospital, Department of Surgery, Center for Surgery and Public Health, Boston, USA, 9 Harvard Medical School \& Harvard T.H. Chan School of Public Health, Boston, USA, 10 Hospital Universitario del Valle, Department of Surgery, Division of Trauma and Acute Care Surgery. Cali, Colombia.

\section{Abstract}

Trauma is a complex pathology that requires an experienced multidisciplinary team with an inherent quick decision-making capacity, given that a few minutes could represent a matter of life or death. These management decisions not only need to be quick but also accurate to be able to prioritize and to efficiently control the injuries that may be causing impending hemodynamic collapse. In essence, this is the cornerstone of the concept of damage control trauma care. With current technological advances, physicians have at their disposition multiple diagnostic imaging tools that can aid in this prompt decision-making algorithm. This manuscript aims to perform a literature review on this subject and to share the experience on the use of whole body computed tomography as a potentially safe, effective and efficient diagnostic tool in cases of severely injured trauma patients regardless of their hemodynamic status. Our general recommendation is that, when feasible, perform a whole body computed tomography without interrupting ongoing hemostatic resuscitation in cases of severely injured trauma patients with or without signs of hemodynamic instability. The use of this technology will aid in the decision-making of the best surgical approach for these patients without incurring any delay in definitive management and/or increasing significantly their radiation exposure. 
Copyright: (c) 2020 Universidad del Valle.

(1) $(20$

Conflict of Interest:

Authors have not any conflict of interest

Corresponding author:

Carlos A. Ordonez, MD, FACS.

Division of Trauma and Acute Care

Surgery, Department of Surgery.

Fundación Valle del Lili. Cali, Colombia

Division of Trauma and Acute Care

Surgery, Department of Surgery,

Universidad del Valle, Cali, Colombia;

Universidad Icesi, Cali, Colombia.

Email: ordonezcarlosa@gmail.com,

carlos.ordonez@fvi.org.co

\section{Resumen}

El trauma es una compleja patología que requiere un equipo experimentado y multidisciplinario con una capacidad para la toma de decisiones Oportuna ya que en unos pocos minutos pueden representar la diferencia entre la vida y la muerte. Estas decisiones deben ser precisas para ser capaces de priorizar y controlar eficientemente las lesiones que puedan estar causando el compromiso hemodinámico. En esencia, este es el punto clave del concepto de control de daños en la atención del trauma. Con los nuevos avances tecnológicos, el equipo médico tiene a disposición múltiples herramientas imagenológicas de diagnóstico. Este artículo presenta una revisión de la literatura y descripción de la experiencia local con el uso de la tomografía corporal total como una herramienta diagnostica potencialmente segura, efectiva y eficiente en casos de pacientes con trauma severo sin importar su estado hemodinámico. La recomendación general, cuando sea posible, es que se debe realizar una tomografía corporal total sin interrumpir las maniobras de resucitación hemostática en casos de pacientes severamente traumatizados con o sin signos de inestabilidad hemodinámica. El uso de esta tecnología tiene como objetivo tomar decisiones pertinentes y definir el mejor abordaje quirúrgico para el paciente sin incurrir en tardanzas en el manejo definitivo o incrementar el tiempo de exposición a la radiación.

\section{Remark}

\section{1) Why was this study conducted?}

Analysis the use of whole-body tomography as a diagnostic tool potentially safe, effective, and feasible for a severe trauma patient with or without hemodynamic instability

\section{2) What were the most relevant results of the study?}

The use of whole-body tomography can be performed without stop the resuscitation maneuvers for severe trauma patients

\section{3) What do these results contribute?}

The use of whole-body tomography in the initial survey allows the appropriate making decisions to define the best surgical approach without delays in the definitive management. 


\section{Background}

Trauma is a complex pathology that requires an experienced multidisciplinary team with an inherent quick decision-making capacity, given that a few minutes could represent a matter of life or death ${ }^{1}$. These management decisions not only need to be quick but also accurate to be able to prioritize and to efficiently control the injuries that may be causing impending hemodynamic collapse. In essence, this is the cornerstone of the concept of damage control trauma care. With current technological advances, physicians have at their disposition multiple diagnostic imaging tools that can aid in this prompt decision-making algorithm. This manuscript aims to perform a literature review on this subject and to share the experience from the Trauma and Emergency Surgery Group of Cali, Colombia on the use of whole body computed tomography as a potentially safe, effective and efficient diagnostic tool in cases of severely injured trauma patients regardless of their hemodynamic status.

This article is a consensus that synthesizes the experience earned during the past 30 years in trauma critical care management of the severely injured patient from the Trauma and Emergency Surgery Group of Cali, Colombia which is made up of experts from the University Hospital Fundación Valle del Lili, the University Hospital del Valle "Evaristo García", the Universidad del Valle and Universidad Icesi, the Colombian Association of Surgery, the Pan-American Trauma Society and the collaboration of international specialists of the United States of America.

\section{Diagnostic imaging during the initial evaluation of a trauma patient}

Management of blunt or penetrating hemodynamically unstable trauma patients should be initially focused on an early and aggressive hemostatic resuscitation, prompt diagnosis of all life-threatening injuries, and early control of ongoing surgical bleeding. The advanced trauma life support manual provides a useful guide on how to systematically evaluate the polytraumatized patient. During the primary survey, resuscitation is initiated simultaneously while the overall physical examination is being performed. This initial physical examination does not always reveal the source of the patient's hemodynamic instability ${ }^{2}$. The goal then is to identify potential life-threatening injuries in order to treat them promptly by using adjuvants such as a portable chest and pelvic X-ray and a focused abdominal sonography for trauma as proposed by the manual. The preface on the use of these basic diagnostic tools is that they do not delay the onset of definitive management and/or ongoing resuscitation ${ }^{3}$. The use of these diagnostic aids provides valuable information that can help guide in choosing the optimal surgical management of these patients ${ }^{4,5}$. X-Rays and the focused abdominal sonography for trauma, although useful adjuncts, also are self-limiting per se due to their intrinsic sensitivity and specificity to identify accurately all life-threatening injuries. Computed tomography has emerged as a highly sensitive and specific diagnostic tool and an integral component of the primary evaluation of trauma patients in many centers around the world ${ }^{6-9}$. However, the use of computed tomography has been associated with time delays between the emergency department and the operating room and the potential increase of radiation exposure that could in turn increase the long-term risk of cancer ${ }^{6}$.

\section{Organ selective computed tomography versus whole body computed tomography}

Organ selective computed tomography are scans that are targeted to characterize specific injuries suspected during initial physical exam and/or initial adjuvants performed by the treating physician. On the contrary, whole body computed tomography are scans that include the brain, chest, abdomen, pelvis, and the cervical, thoracic and lumbar spine. They are included in the primary evaluation of a trauma patient following a pre-established institutional protocol which in turn negates the potential human error on behalf of the treating physician and it also has the intrinsic 
capability of identifying less evident injuries that may be missed with plain X-rays and focused abdominal sonography for trauma exam. All of these technical virtues can potentially improve overall prognosis and decrease waiting times in the emergency department ${ }^{9-11}$. To validate these assertions, we performed a retrospective evaluation of all severely injured patients at our Level I Trauma center in Cali, Colombia from January 2016 to December 2017. We compared the radiation exposure and the time between emergency department and operating room between patients who received organ selective computed tomography versus those who received a whole body computed tomography. A total of 123 patients were included, 53 received organ selective computed tomography and 70 whole body computed tomography. Forty seven percent of patients from the organ selective computed tomography Group required subsequent additional computed tomography's to rule out suspected end organ injuries. In contrast, patients in the whole body computed tomography Group did not require any additional computed tomography's. Median radiation exposure dose in the organ selective computed tomography Group was $22 \mathrm{mSv}$ (IQR= 6-31) and in the whole body computed tomography Group it was $15.1 \mathrm{mSv}(\mathrm{IQR}=9.9-24.8)$ $(p<0.001)$. Furthermore, the time between image acquisition and diagnosis was lower in the whole body computed tomography Group [ $22 \min (\mathrm{IQR}=14-32)$ vs. $32 \min (21-65) ; p<0.001]^{12}$. With these results and supporting evidence obtained from multiple previous studies, we can conclude that whole body computed tomography is an efficient and safe tool for the early diagnosis of severely injured trauma patients. Beyond these conclusions we are also finding a large body of evidence supporting its use in regards to overall survival benefits and cost-efficiency ${ }^{8,13-16}$.

\section{Implementing whole body computed tomography protocols}

Worldwide, there are many whole body computed tomography protocols with no real consensus on which one is the best ${ }^{10,17-20}$. Most of them include acquisition without intravenous contrast for the brain and cervical spine, followed by a two-pass contrasted technique for the chest, abdomen, and pelvis. The duration of the acquisition of these images typically varies between 2 and 5 minutes. As we mentioned before, the radiological evaluation of the severely injured trauma patient must be quick without interrupting ongoing resuscitation and at the same time minimizing the patient's exposure to radiation. Also, keep in mind that both arterial and venous phases must be obtained to properly evaluate end-organ and/or vascular injuries ${ }^{21,22}$. To this end, we have implemented at our Trauma Center in Cali, Colombia the use of whole body computed tomography for all severely injured trauma patients regardless of their hemodynamic status. We have done this via a single-pass whole body computed tomography protocol in which the acquisition time is 175 seconds (2.92 minutes), and consist of a simple non-contrast brain phase, followed by a single-pass from neck to pubic symphysis with contrast (Table 1).

Table 1. Single-Pass WBCT Protocol 26

PHASE A Simple Acquisition Phase: Brain

Contrast Administration Phase: chest, abdomen, pelvis, and cervical, thoracic and lumbar spine

IV Contrast: iodine-based, non-ionic hypo-osmolar $(370 \mathrm{mg} / \mathrm{mL})$

Flow rate $=2 \mathrm{cc} / \mathrm{s}$

Step 1. First Injection

Volume Contrast $=60 \mathrm{cc}$

Pause $=45 \mathrm{~s}$

Total Time $=75 \mathrm{~s}$

PHASE B

Flow rate $=4 \mathrm{cc} / \mathrm{s}$

Step 2. Second Injection Volume Contrast $=60-70 \mathrm{cc}$ Flush $=40 \mathrm{cc}$ Normal Saline Total Time $=100 \mathrm{~s}$

Step 3. Contrasted Acquisition In the Descending Aorta ROI $=200$ $\mathrm{HU}$ after the second injection

IV: Intravenous; ROI: Region of Interest; HU: Hounsfield Units 


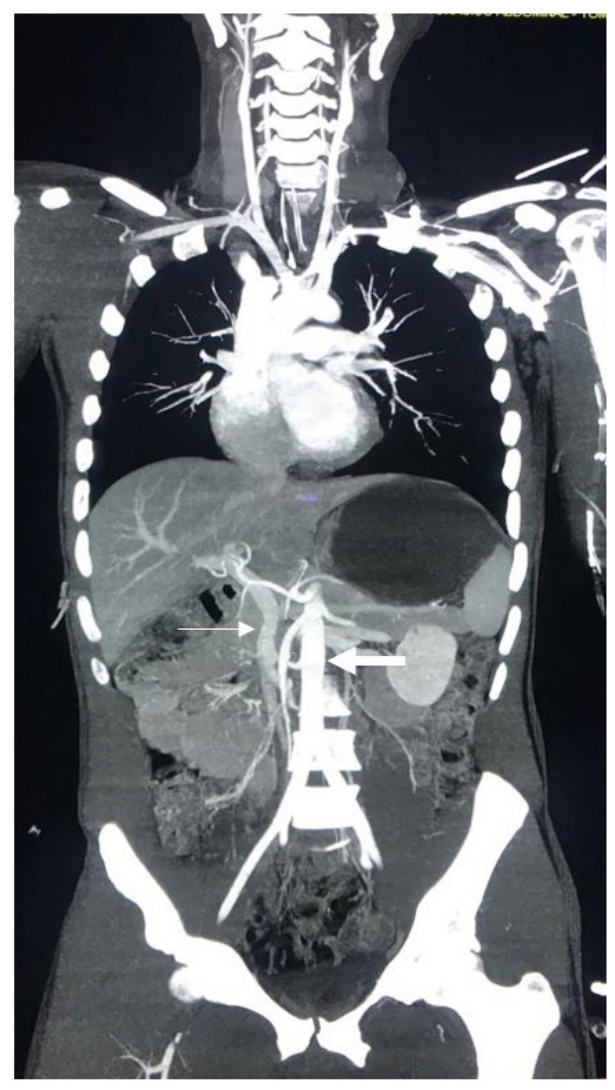

Figure 1. Arterial and venous phases on whole body computed tomography (the bold arrow shows arterial phase and the lighter arrow shows venous phase)

The result is both a venous and arterial phase in a single high-resolution image (Figure 1). From January 2017 to December 2018, an observational, prospective study was performed at our Level I Trauma Center where we tabulated a total of 263 severely injured trauma patients who underwent whole body computed tomography as part of their initial evaluation. One hundred and sixty-eight had blunt trauma and were hemodynamically stable (Group 1), 50 patients had blunt trauma and were hemodynamically unstable (Group 2) and 45 had penetrating trauma with or without hemodynamic instability (Group 3). Median injury severity score was $22(\mathrm{IQR}=16-22)$ and $172(65.4 \%)$ patients received non-operative management, without significant differences between groups. Median time between emergency department and whole body computed tomography was 28 minutes (IQR= 14-5) and the median radiation dose was less than $20 \mathrm{mSv}$, which is considered to be the optimal range of exposure that does not increase significantly long-term cancer risks ${ }^{23-25}$. Ninety-one (34\%) patients required surgical management, without significant differences among groups [Group 1: 59 (35.1\%); Group 2: 15 (30\%); Group 3: 17 (37.7\%); $p=0.23$ ]. All patients who required surgery had positive findings during their procedure ${ }^{26}$. These results provide supporting evidence towards our claims that whole body computed tomography is a safe, effective and efficient tool in the initial workup and subsequent management of the severely injured trauma patient regardless of their hemodynamic status. Furthermore, it could potentially avoid unnecessary procedures in patients that could be managed otherwise nonoperatively, decreasing overall costs and morbidity in these patients. 


\section{Whole body computed tomography is safe, effective and efficient}

Many trauma surgeons believe in the utility of whole body computed tomography in the initial evaluation of trauma patients but emphasize that its use should be restricted only to those patients that are hemodynamically stable and those that are not hemodynamically stable should be taken immediately to the operating room /angiography suite. However, it is not always possible to determine exactly in the emergency department the source of the patient's hemodynamic instability especially in cases of multiple penetrating or blunt trauma. To this end, it would not be practical to explore multiple cavities from the neck to the pelvis to control all sources of injury. In these cases, the use of whole body computed tomography is essential to plan the surgical intervention and to direct and prioritize the efforts towards controlling all life-threatening injuries and significant surgical ongoing bleeding. In a previous retrospective evaluation of cases at our institution between 2012 and 2013, 171 patients with severe trauma and hemodynamic instability we found that $80(47 \%)$ patients underwent whole body computed tomography upon admission (Group 1) and 91 (53\%) were taken directly to the operating room (Group 2). Of these, whole body computed tomography was helpful in deciding definitive/specific surgical management in 46\%. Forty-three (54\%) patients from the whole body computed tomography Group received non-operative management and none of them died or had any complications during whole body computed tomography. There were no differences regarding overall mortality between the groups [Group 1:10 (12.5\%); Group 2: 16 $(17.6 \%) ; p=0.23]^{27}$.

Our findings are consistent with those found in the literature where the liberal use of whole body computed tomography has significantly increased the probability of survival of the severely injured trauma patient ${ }^{17}$. An example of those studies, is the one published by HuberWagner et al. In this study a total of 3,924 patients underwent computed tomography as part of their initial trauma workup and of these 2,430 had organ selective computed tomography and 1,494 whole body computed tomography. The predicted mortality when compared to the actual mortality rate among both groups was found to be significantly higher in the whole body computed tomography Group $(23.2 \%$ vs. $17.3 \%$; $p<0.001)$ than in the organ selective computed tomography Group ( $17.1 \%$ vs. $17.5 \% ; p=0.66){ }^{13}$. These findings where expanded on a follow-up study in 2013 by the same authors which included a total of 16,719 patients (9,233 whole body computed tomography Group; 7,486 organ selective computed tomography Group) and found that the absolute mortality rate in the whole body computed tomography Group was significantly lower than that of the organ selective computed tomography Group $(17.4 \% \text { vs. } 21.4 \% ; p<0.001)^{11}$. Several meta-analyses have confirmed these findings and the overall concept that whole body computed tomography is a safe, effective, and efficient surgical triage tool ${ }^{6,9,28}$. To this point, the inclusion of a whole body computed tomography protocol in the initial management of the severely injured trauma patient has been seen to decrease the time to definitive diagnosis, increase the accuracy of patient selection between operative vs. non-operative management, and reduce the overall radiation exposure.

Therefore, whole body computed tomography has evolved worldwide to became an intrinsic component of the early diagnosis and management of the severely injured ${ }^{12,17,26}$.

\section{Conclusion}

Our general recommendation is that, when feasible, perform a whole body computed tomography without interrupting ongoing hemostatic resuscitation in cases of severely injured trauma patients with or without signs of hemodynamic instability. The use of this technology will aid in the decision-making of the best surgical approach for these patients without incurring any delay in definitive management and/or significantly increasing their radiation exposure. 
Whole-body computed tomography is safe, effective and efficient in the severely injured hemodynamically unstable trauma patient.

\section{References}

1. Gondek S, Schroeder ME, Sarani B. Assessment and resuscitation in trauma management. Surg Clin North Am. 2017; 97: 985-98. doi:10.1016/j.suc.2017.06.001.

2. Arora R, Arora AJ. Justification of whole-body CT in polytrauma patients, can clinical examination help selecting patients? Quant Imaging Med Surg. 2019; 9: 636-41. doi:10.21037/qims.2019.04.02.

3. ATLS Subcommittee Group, Committee American College of Surgeons', International ATLS working. Advanced trauma life support (ATLS(r)): The ninth edition. J Trauma Acute Care Surg. 2013; 74: 1363-6. doi:10.1097/TA.0b013e31828b82f5.

4. Soto JA, Anderson SW. Multidetector CT of blunt abdominal trauma. Radiology. 2012; 265: 678-93. doi:10.1148/radiol.12120354.

5. Wada D, Nakamori Y, Yamakawa K, Yoshikawa Y, Kiguchi T, Tasaki O, et al. Impact on survival of whole-body computed tomography before emergency bleeding control in patients with severe blunt trauma. Crit Care. 2013; 17: 1-7. doi:10.1186/cc12861.

6. Jiang L, Ma Y, Jiang S, Ye L, Zheng Z, Xu Y, et al. Comparison of whole-body computed tomography vs selective radiological imaging on outcomes in major trauma patients: A meta-analysis. Scand J Trauma Resusc Emerg Med. 2014; 22: 1-11. doi:10.1186/s13049-014-0054-2.

7. Dreizin D, Munera F. Multidetector CT for Penetrating Torso Trauma: State of the Art. Radiology. 2015; 227: 338-55. doi:10.1017/CBO9781107415324.004.

8. Kinoshita T, Yamakawa K, Matsuda H, Yoshikawa Y, Wada D, Hamasaki T, et al. The Survival Benefit of a Novel Trauma Workflow that Includes Immediate Whole-body Computed Tomography, Surgery, and Interventional Radiology, All in One Trauma Resuscitation Room: A Retrospective Historical Control Study. Ann Surg. 2019; 269: 370-6. doi:10.1097/SLA.0000000000002527.

9. Chidambaram S, Goh EL, Khan MA. A meta-analysis of the efficacy of whole-body computed tomography imaging in the management of trauma and injury. Injury. 2017; 48: 1784-93. doi:10.1016/j.injury.2017.06.003.

10. Smith CM, Woolrich-Burt L, Wellings R, Costa ML. Major trauma CT scanning: The experience of a regional trauma centre in the UK. Emerg Med J. 2011; 28: 378-82. doi:10.1136/emj.2009.076414.

11. Huber-Wagner S, Biberthaler P, Häberle S, Wierer M, Dobritz M, Rummeny E, et al. Whole-Body CT in Haemodynamically Unstable Severely Injured Patients - A Retrospective, Multicentre Study. PLoS One. 2013; 8(7): e68880. doi:10.1371/journal.pone.0068880.

12. Ordoñez C, del Valle AM, Parra M, Guzman-Rodriguez M, Herrera-Escobar JP, García C, et al. Singlepass Whole-body vs Organ-selective Computed Tomography for Trauma: Timely Diagnosis vs Radiation Exposure: An Observational Study. Panam J Trauma Crit Care Emerg Surg. 2020; 9(1): 26-31. Doi: 10.5005/ jp-journals-10030-1262.

13. Huber-Wagner S, Lefering R, Qvick LM, Körner M, Kay M V., Pfeifer KJ, et al. Effect of whole-body CT during trauma resuscitation on survival: a retrospective, multicentre study. Lancet. 2009; 373: 1455-61. doi:10.1016/S0140-6736(09)60232-4.

14. Tsutsumi Y, Fukuma S, Tsuchiya A, Ikenoue T, Yamamoto Y, Shimizu S, et al. Computed tomography during initial management and mortality among hemodynamically unstable blunt trauma patients: A nationwide retrospective cohort study. Scand J Trauma Resusc Emerg Med. 2017; 25: 1-8. doi:10.1186/s13049-017-0396-7. 
15. Hutter M, Woltmann A, Hierholzer C, Gärtner C, Bühren V, Stengel D. Association between a single-pass whole-body computed tomography policy and survival after blunt major trauma: A retrospective cohort study. Scand J Trauma Resusc Emerg Med. 2011; 19: 73. doi:10.1186/1757-7241-19-73.

16. Kimura A, Tanaka N. Whole-body computed tomography is associated with decreased mortality in blunt trauma patients with moderate-to-severe consciousness disturbance: A multicenter, retrospective study. J Trauma Acute Care Surg. 2013; 75: 202-6. doi:10.1097/TA.0b013e3182905ef7.

17. Çorbacioglu SK, Aksel G. Whole body computed tomography in multi trauma patients: Review of the current literature. Turkish J Emerg Med. 2018; 18: 142-7. doi:10.1016/j.tjem.2018.09.003.

18. Godt JC, Eken T, Schulz A, Johansen CK, Aarsnes A, Dormagen JB. Triple-split-bolus versus single-bolus CT in abdominal trauma patients: a comparative study. Acta Radiol. 2018; 59: 1038-44. doi:10.1177/0284185117752522.

19. Leidner B, Adiels M, Aspelin P, Gullstrand P, Wallén S. Standardized CT examination of the multitraumatized patient. Eur Radiol. 1998; 8: 1630-8. doi:10.1007/s003300050601.

20. Hickethier T, Mammadov K, Baeßler B, Lichtenstein T, Hinkelbein J, Smith L, et al. Whole-body computed tomography in trauma patients: Optimization of the patient scanning position significantly shortens examination time while maintaining diagnostic image quality. Ther Clin Risk Manag. 2018; 14: 849-59. doi:10.2147/TCRM. S162074.

21. Hakim W, Kamanahalli R, Dick E, Bharwani N, Fetherston S, Kashef E. Trauma whole-body MDCT: An assessment of image quality in conventional dual-phase and modified biphasic injection. Br J Radiol. 2016; 89(1063):20160160. doi:10.1259/bjr.20160160.

22. Boscak AR, Shanmuganathan K, Mirvis SE, Fleiter TR, Miller LA, Sliker CW, et al. Optimizing trauma multidetector CT protocol for blunt splenic injury: Need for arterial and portal venous phase scans. Radiology. 2013; 268: 79-88. doi:10.1148/radiol.13121370.

23. Brenner DJ, Elliston CD. Estimated Radiation Risks Potentially Associated with Full-Body CT Screening. Radiology. 2004; 232: 735-8.

24. Pearce MS, Salotti JA, Little MP, McHugh K, Lee C, Kim KP, et al. Radiation exposure from CT scans in childhood and subsequent risk of leukaemia and brain tumours: A retrospective cohort study. Lancet. 2012; 380: 499-505. doi:10.1016/S0140-6736(12)60815-0.

25. McCollough CH, Schueler BA. Calculation of effective dose. Am Assoc Phys Med. 2000; 27: 828-37. doi:10.1118/1.598948.

26. Ordoñez CA, Holguín A, García C, Parra MW, Angamarca E, Guzmán-Rodríguez M, et al. Implementation of a new Single-Pass Whole-Body Computed Tomography Protocol: Is it safe, effective and efficient in patients with severe trauma?. Colomb Med (Cali). 2020; 51(1): e4224.

27. Ordoñez CA, Herrera-Escobar JP, Parra MW, Rodriguez-Ossa PA, Mejia DA, Sanchez Al, et al. Computed tomography in hemodynamically unstable severely injured blunt and penetrating trauma patients. J Trauma Acute Care Surg. 2016; 80: 597-603. doi:10.1097/TA.0000000000000975.

28. Caputo ND, Stahmer C, Lim G, Shah K. Whole-body computed tomographic scanning leads to better survival as opposed to selective scanning in trauma patients: A systematic review and meta-analysis. J Trauma Acute Care Surg. 2014; 77: 534-9. doi:10.1097/TA.0000000000000414. 